Rechtsvergleichende Analyse 


\title{
Die Alterssicherung der Beamten im Rechtsvergleich
}

\author{
Peter A. Köhler
}

I. Die Rechtslage in den Vergleichsländer

1. Gibt es ein Beamtenstatut?

2. Dienstrecht und Altersversorgung

3. Reformen

II. Die Rechtslage in den Internationalen Organisationen des VN-Systems und der EU 1. Das Beamtenstatut der ,internationalen Beamten“"

2. Altersversorgung

3. Reformen

III. Auswertung

1. Beamtentum als Institution des Verfassungsrechts

2. Alimentation und Alterssicherung

3. Die Wahrnehmung hoheitlicher Aufgaben

4. Ein besonderes Beamtenethos gilt nur für besondere Funktionen - „Das Amt macht den Beamten“

5. Ergebnis 
Ziel des nachstehenden Rechtsvergleichs ist es, die Antwort auf mehrere, aufeinander bezogene Fragen zu finden: Folgt aus der Besonderheit des Dienstherrn (Staat, internationale oder supranationale Organisation) eine Besonderheit der Aufgaben (Betrauung mit einem Amt, hoheitliche Tätigkeit)? Begründet dies eine Besonderheit des Dienstrechts? Ist daraus wiederum eine besondere soziale Sicherung im Alter abzuleiten?

Zuerst soll deshalb geprüft werden, ob sich in den zum Vergleich herangezogenen nationalen Rechtsordnungen ein Sonderstatus für bestimmte, in der öffentlichen Verwaltung beschäftigte Personen feststellen läßt. Dabei - und entsprechend für das Dienstrecht von Europäischer Union und Vereinten Nationen - wird nach dem „Beamtenstatut" gefragt, also nach dem Recht, das gegebenenfalls diesen Status festlegt und charakterisiert.

Danach wird geprüft, ob dieser Status auch nach Beendigung des Dienstes für die Ausgestaltung der Versorgung im Alter bestimmend wird. Schließlich wird dargestellt, inwieweit derartige Sonderformen der Altersversorgung unmittelbar das Ziel von Reformen waren oder ob sie mittelbar von Reformen des jeweiligen allgemeinen Alterssicherungssystems tangiert worden sind. Dabei bildet die im Landesbericht Deutschland beschriebene Rechtslage quasi die „Diskussionsgrundlage“, vor der Ähnliches und Unterschiedliches erkennbar und vergleichbar wird.

\section{Die Rechtslage in den Vergleichsländer}

\section{Gibt es ein Beamtenstatut?}

Im Beamtenrecht der Nachbarländer Deutschland und Frankreich, das auch für die Gestaltung des Dienstrechts der EU bestimmend war, finden sich weitgehende prinzipielle Parallelen, aber auch eine Reihe bedeutsamer Unterschiede.

So wird in Frankreich der Beamtenstatus durch gesetzlich geregelte Ernennung und Zuweisung eines Amtes auf Lebenszeit erlangt. Nach der Verfassung sind Rechte und Pflichten der Beamten vom Gesetzgeber festzulegen. Die Beamtenlaufbahn endet gewöhnlich mit dem Eintritt in den Ruhestand. Anders als in Deutschland haben die Beamten (mit Ausnahme z.B. von Polizei) das Recht zu streiken, eingeschränkt allerdings durch das beamtenrechtliche Gebot der Zurückhaltung und unter Aufrechterhaltung der wesentlichen Aufgaben des öffentlichen Dienstes.

Die italienische Verfassung erwähnt zwar die Beamten - allerdings nur hinsichtlich des ordnungsgemäßen, unparteiischen und effizienten Funktionierens der Verwaltung. Eine statusrechtliche Sonderstellung der Beamten als Teil des öffentlichen Dienstes wird verfassungsrechtlich nicht vorgegeben. Das einfachgesetzliche Dienstrecht regelt allein die dem öffentlichen Recht unterliegenden Dienstverhältnisse. Es gilt nur für eine 
begrenzte Gruppe von Bediensteten mit besonderen hoheitlichen Aufgaben wie Richter, Staatsanwälte, Leitungspersonal im Strafvollzug. Für diese Gruppe kann im funktionalen Sinn von einem „beamtenähnlichen Status“ nach deutschem Rechtsverständnis gesprochen werden.

Demgegenüber finden sich zur Schweiz diametrale Gegensätze: Während in Deutschland die Rechtstellung der Beamten verfassungsrechtlich festgeschrieben ist, gibt es in der schweizerischen Bundesverwaltung seit den Reformen der 1990er Jahre die Rechtsposition „Beamte“ nicht mehr: Das Bundespersonal besteht aus Angestellten, was auch für das auf Zeit gewählte Bundespersonal wie etwa Staatsanwälte des Bundes gilt. Einige Pflichten, etwa die besondere Treuepflicht, erinnern noch an den Beamtenstatus von früher; doch sonst (z.B. befristete oder unbefristete Beschäftigungsverhältnisse und deren Beendigung durch Kündigung sind möglich, die Entlohnung erfolgt nach dem Leistungsprinzip) unterscheidet sich das Dienstrecht des Bundespersonals kaum noch von dem des Personals in der Privatwirtschaft.

Dem ähnlich ist die Rechtstellung der civil servants im Vereinigten Königreich, das ebenfalls kein spezifisches „Beamtenrecht“ kennt. Betrachtet man die Situation im Vereinigten Königreich funktional, ändert sich das Bild etwas: Es finden sich einige der Kriterien wieder, wie sie vom Bundesverfassungsgericht in Deutschland zur Bestimmung der Grundsätze des Berufsbeamtentums herausgearbeitet worden sind. So unterliegen die civil servants der Prärogative der Krone, der gegenüber sie zu Loyalität und Verschwiegenheit verpflichtet sind. Sie üben hoheitliche Tätigkeiten aus. Bestimmte Dienste setzen deshalb die Staatsangehörigkeit des Vereinigten Königreichs voraus.

In Dänemark sind die Dienstverhältnisse bei Staat und Kommunen aufgrund der Verfassung gesetzlich geregelt. Die Hauptpflicht der Beamten besteht in der korrekten Ausübung des Dienstes, es besteht ein Streikverbot.

In Norwegen gelten für eine herausgehobene Gruppe von Beamten (= embetsmen) bestimmte Vorgaben der Verfassung, das allgemeine Dienstrecht ist gesetzlich geregelt. Die Staatsbediensteten werden danach grundsätzlich unbefristet eingestellt. Es gibt eine Disziplinarordnung und einen besonderen Rechtsweg für dienstliche Beschwerden.

Die Verfassung von Schweden regelt einige Prinzipien des Beamtenrechts, wonach u.a. die für die Rechtstellung der Bediensteten bei Staat und Kommunen grundlegenden Bestimmungen ebenfalls durch Gesetz getroffen werden müssen. Dieses enthält eine „Disziplinarordnung“, Vorschriften für regelmäßige Gesundheitsuntersuchungen sowie Regeln für das Verhalten bei Arbeitskonflikten. Die Bedingungen zur Aufnahme von Nebentätigkeiten sind genau geregelt. Zur Beendigung von Dienstverhältnissen gibt es einige, das allgemeine arbeitsrechtliche Kündigungsschutzgesetz ergänzende Regeln.

Der Staatsdienst in der Russischen Föderation wurde in bewußter Abkehr vom System der Sowjetunion neu gestaltet. Viele Einzelheiten sind noch im Fluß. Die Verfassung gibt als Ausdruck des Demokratieprinzips allein den gleichen Zugang aller Bürger zum Staatsdienst vor. Dieser selbst ist gesetzlich sehr detailliert geregelt. Das Fehlen u.a. der Normierung einer besonderen Treuepflicht oder des Lebenszeit- und des Ali- 
mentationsprinzips erklärt sich aus dem „Mischcharakter“ des Dienstrechts: Es folgt, wenn das Gesetz nichts anderes vorschreibt, dem allgemeinen Arbeitsrecht, es hat also sowohl öffentlich-rechtlichen wie arbeitsrechtlichen Charakter.

Auch die slowenische Verfassung regelt den Zugang zum Verwaltungsdienst durch öffentliche Ausschreibung. In Übereinstimmung mit dem europäischen Recht und der Rechtsprechung des EuGH umfaßt der öffentliche Dienst die Beschäftigung in einem Amt mit hoheitlichen Befugnissen. Das Dienstrecht der slowenischen Beamten ist von einem ähnlichen „Mischcharakter“ geprägt wie in der Russischen Föderation: Nach der „monistischen Konzeption des Arbeitsrechts“ sind die in Sondergesetzen für bestimmte Beamte (z.B. Polizei, Justiz) enthaltenen Prinzipien lex specialis. Dazu gehören u.a. das Legalitätsprinzip, Professionalität oder die Pflicht zur Amtsverschwiegenheit. Dennoch wird das Beamtenverhältnis nicht als besonderes öffentlich-rechtliches Dienstverhältnis verstanden.

In der Tschechischen Republik ist das Dienstrecht der Beamten im allgemein gültigen Arbeitsgesetzbuch geregelt. Darin sind bestimmte Pflichten (z.B. Amtsverschwiegenheit, Einschränkung von Nebenbeschäftigungen) statuiert. Es fehlt aber eine - etwa verfassungsrechtlich begründete - besondere Rechtstellung. Zwar ist für den Staatsdienst ein besonderes Dienstgesetz ausgearbeitet worden, es ist aber bislang nicht in Kraft getreten.

\section{Dienstrecht und Altersversorgung}

In Deutschland bestimmen das Alimentationsprinzip und das Lebenszeitprinzip als Grundprinzipien des Beamtenrechts unmittelbar auch das Recht der Altersversorgung von Beamten. Diese Versorgung stellt ein Sondersystem neben der gesetzlichen Rentenversicherung dar. Sie muß ,amtsangemessen“ sein, d.h. sie wird (neben einem Zeitund Wertfaktor) wesentlich vom zuletzt innegehabten Amt bestimmt. Das Altersruhegehalt kann derzeit höchstens 71,75 \% des letzten Gehalts erreichen. Aus dem Alimentationsprinzip folgt zudem ein Mindestversorgungsanspruch sowie die Beitragsfreiheit der Beamten: Die Finanzierung der Beamtenversorgung erfolgt allein durch den Dienstherrn aus Steuermitteln.

In Frankreich wird die Altersversorgung der Beamten durch Sondersysteme unmittelbar vom Staat getragen. Der Ruhestand wird als Fortsetzung der aktiven Arbeitszeit verstanden. Die Höhe der Pension korrespondiert mit der hierarchischen Stellung des Beamten sowie mit der Dauer und der Art des Dienstes. Liegen die gesetzlichen Voraussetzungen vor, so beträgt die Pension bis zu $75 \%$ der während der letzten sechs Monate vor Eintritt in den Ruhestand erreichten Bezüge. Am Ende der Laufbahn werden die dem ausgeübten Dienst angemessenen materiellen Voraussetzungen garantiert.

Obwohl für die Bediensteten mit einem öffentlich-rechtlich ausgestalteten Dienstverhältnis in Italien funktional von einem beamtenähnlichen Status gesprochen werden 
kann, verknüpft das Recht diesen speziellen dienstrechtlichen Status nicht mehr mit Besonderheiten bezüglich der Alterssicherung dieser Personengruppen. Die Alterssicherung dieser Bediensteten wurde wie die des öffentlichen Dienstes insgesamt den Regelungen der allgemeinen gesetzlichen Rentenversicherung angeglichen. Die italienische Verfassung enthält keine Vorgaben zum Schutz der Berufsbeamten, die diesem Harmonisierungsprozess der Alterssicherung entgegenstünden.

Die Altersversorgung des Bundespersonals der Schweiz ist in die 1. und 2. Säule der allgemeinen Alterssicherung integriert. Die Regeln der Alters- und Hinterlassenversicherung (1. Säule) kennen keine Sondertatbestände für das Bundespersonal. Dem Verfassungsauftrag gemäß sichert die 1. Säule eine Mindestrente nach Erreichen einer bestimmten Anzahl von Beitragsjahren. Die Beiträge werden vom Arbeitnehmer und vom Arbeitgeber zur Hälfte getragen, wegen der Existenzsicherungsfunktion übernimmt der Staat einen Teil der Finanzierung. Für das Bundespersonal hat die betriebliche Vorsorge (2. Säule) eine besondere Bedeutung, weil sie für Sonderregeln offen ist. Die frühere Privilegierung des Bundespersonals durch Orientierung der Pensionen am letzten Gehalt wurde aufgehoben, die Pensionen folgen inzwischen den Altersgutschriften pro Beitragsjahr. Da das letzte Gehalt dabei prozentual aber höher angerechnet wird, ergibt sich dennoch eine, wenn auch im Effekt verminderte, „Quasi-Orientierung“ am zuletzt bezogenen Gehalt. Altersleistungen können Renten und Kapitalabfindung sein. Die Beiträge, die zur Altersgutschrift für das Bundespersonal führen, übertreffen die Mindestvorgaben des Gesetzes, u.a. weil der Arbeitgeber (Bund) stets mehr als deren Hälfte übernimmt. Die Kapitalabfindung, die sich ebenfalls an der Altersgutschrift orientiert, kann beim Großteil des Bundespersonals in Höhe von bis zu $100 \%$ ausgezahlt werden. Damit wird das Risiko der Verwendung dieses Kapitals in die Hände der Angestellten gelegt, was aufgrund der Existenzsicherung durch die 1. Säule akzeptiert wird. Das Anlageverhalten während des Vermögensaufbaus durch die Einrichtungen der beruflichen Vorsorge wird intern wie extern kontrolliert; eine Mindestverzinsung ist vorgegeben. Darüber hinaus werden finanzielle Einbußen von einem eigenständigen Fonds aufgefangen. Schließlich hat der Bund zur Sicherstellung der Finanzierung der 2. Säule für das Bundespersonal beträchtliches Kapital aufgewandt.

Im Vereinigten Königreich ist die Altersversorgung der civil servants in das allgemeine System eingegliedert, soda $\beta$ diese insbesondere die State Basic Pension als Grundsicherung beanspruchen können. Große Bedeutung kommt daneben den diversen Formen der betrieblichen Alterssicherung zu, die bislang primär durch Arbeitgeberbeiträge finanziert wurden und deren Leistungen vom letzten Gehalt vor der Pensionierung bestimmt waren. Seit dem 30. Juli 2007 gilt für neu eingestellte civil servants das betriebliche Versorgungssystem „nuvos“, ein defined benefits scheme, für das mit jedem Dienstjahr 2,3\% des pensionsbegründenden Gehalts als Basis für die spätere Pension erworben werden. Diese deutlich erhöhte jährliche Quote soll die Abkehr vom Endgehalt als Berechnungsgrundlage der Altersrente ausgleichen. Das Rentenalter wurde von 60 auf 65 Jahre angehoben. Zur Finanzierung tragen die civil servants mit eigenen Bei- 
trägen in Höhe von 3,5 \% des pensionsbegründenden Gehalts bei. Die Beitragshöhe des Arbeitgebers wird so festgelegt, daß sie zusammen mit den Beiträgen der civil servants die Kosten der bislang erworbenen Rentenanwartschaften decken. Sie belaufen sich derzeit auf durchschnittlich 19,4\% des pensionsbegründenden Gehalts. Anstelle der klassischen betrieblichen Alterssicherung besteht für die civil servants die Möglichkeit, Anlagen am Kapitalmarkt (partnership scheme) zu tätigen. Diese Anlageform ist in das umfassende Regulierungssystem für die sog. stake holder pension eingebunden. Für beide Alternativen kann ein Teil der Rente innerhalb bestimmter Grenzen in eine steuerfreie Pauschalzahlung umgewandelt werden, weil die weiterhin als Universalrente geleistete Basic State Pension zu den Renten hinzutritt, die nach Abzug der Kapitalabfindungen verbleiben.

In Dänemark sind die Pensionen für Staats- und Kommunalbeamte gesetzlich geregelt. Die Höhe der Pension wird durch das nach der gesetzlichen Einstufung der Dienststelle erreichte Gehalt zum Zeitpunkt des Eintritts in den Ruhestand und den Dienstjahren des Beamten errechnet. Die höchste Pension beträgt dann $57 \%$ des letzten Gehalts. Die Finanzierung erfolgt für die Staatsbeamten über den Haushalt im Umlageverfahren, für die Kommunalbeamten im kapitalgedeckten System der „Pensionskassen“ der Kommunen.

In Norwegen ist die Alterssicherung der staatlichen und der meisten kommunalen Beamten durch die Staatliche Pensionskasse (SPK), einer ,staatsgarantierten“ Kasse zur Deckung der Pensionskosten gesetzlich geregelt. Obwohl in vielen Details rechtlich mit dem allgemeinen System in Bezug gesetzt, handelt es sich doch um ein Sondersystem. Dafür werden vom Dienstherrn bis zu einer Beitragsbemessungsgrenze $2 \%$ des Gehalts als Beitrag an die SPK einbehalten und abgeführt. Ein Anspruch auf die volle Alterspension in Höhe von 66 \% des zuletzt bezogenen Gehalts setzt 30 Dienstjahre voraus.

In Schweden ist die Alterssicherung der Beamten tarifvertraglich durch den „Pensionsvertrag für Arbeitnehmer beim Staat (PA 03)“ geregelt. Über die Definition von Bemessungsgrenzen ist das Sondersystem mit dem allgemeinen System verbunden. Es sind zwei Pensionsleistungen vorgesehen: die „beitragsbestimmte“ und die „leistungsbestimmte" Alterspension. Für die beitragsbestimmte Alterspension wird ein Beitrag von 2,5\% des Gehalts bis zu einer Bemessungsgrenze an das Staatliche Pensionsamt (SPV) eingezahlt. Der Beamte kann selbst wählen, wie diese Beiträge am Kapitalmarkt angelegt werden sollen. Wird keine Wahl getroffen, werden die Beiträge von SPV in einer traditionellen Versicherung mit Rückzahlungsschutz angelegt. Die leistungsbestimmte Alterspension gilt für Bedienstete mit Gehältern ab einer bestimmten Höhe. Der Pensionsanspruch bestimmt sich nach einem mit der Höhe des Gehalts korrespondierenden unterschiedlichen Prozentsatz. Die volle Leistung setzt 30 Dienstjahre voraus. Die Pensionshöhe errechnet sich aus dem Durchschnittsgehalt der letzten fünf Jahre vor Pensionsantritt. Die Finanzierung der Pensionen erfolgt allein durch den Arbeitgeber, der für die Versicherten die Beiträge bezahlt. Eine von den Arbeitgebern finanzierte kapitalgedeckte Zusatzversicherung hebt das Niveau der Alterssicherung der Beamten. 
In der Russischen Föderation gibt es seit dem Jahr 2002 neben der allgemeinen beitragsfinanzierten „Arbeitsrente“ die steuerfinanzierte staatliche Altersversorgung für die Beamten. Der für das Beamtenstatut festgestellte „Mischcharakter“ findet sich auch in der Altersversorgung wieder: Ein Anspruch auf Pensionsleistungen setzt nämlich das Bestehen von Ansprüchen aus der Arbeitsrente voraus, bei der auch die Staatsbediensteten versichert (und beitragspflichtig) sind. Damit ist die „Beamtenpension“ eher eine Zulage zur allgemeinen Altersversicherung. Ihre Höhe richtet sich u.a. nach den in den letzten zwölf Monaten der Dienstzeit erreichten Durchschnittsbezügen bis zu einer Obergrenze des 2,8-fachen des Grundgehalts.

In Slowenien sind die Beamten in der allgemeinen gesetzlichen Altersversicherung versichert, für Beamte und den öffentlichen Dienst insgesamt ist eine kollektive Zusatzversicherung eingerichtet.

In der Tschechischen Republik sind die Beamten wie alle anderen erwerbstätigen Personen in der gesetzlichen Rentenversicherung versichert. Als einzige Besonderheit sind nach Ablauf bestimmter Dienstzeiten Zulagen vorgesehen.

\section{Reformen}

Die Alterssicherungssysteme aller Vergleichsländer hatten und haben aus den demographischen Entwicklungen die nachfolgend dargestellten Finanzierungsprobleme zu bewältigen, was die Beamtenversorgung nicht unberührt lassen konnte.

In Deutschland wird der gesetzlich vorgeschriebene Aufbau einer Versorgungsrücklage in der Zeit von 2011 bis 2017 dazu führen, daß die Besoldungs- und Versorgungsanpassungen jährlich um $0,2 \%$ niedriger ausfallen werden. Für Neueinstellungen ab dem 1. Januar 2007 wurde auf Bundesebene zur Finanzierung künftiger Versorgungsausgaben zudem ein Versorgungsfonds errichtet, in den der jeweilige Dienstherr regelmäßige Zuweisungen leisten muß. Die Absenkung des Versorgungsniveaus ist nach der Rechtsprechung des Bundesverfassungsgerichts zulässig, sofern der Gesetzgeber insbesondere die Bifunktionalität der Beamtenversorgung beachtet, d.h. der Doppelcharakter der Beamtenversorgung als Verbundsystem aus qualifizierter Grundsicherung und zusätzlicher betrieblicher Altersversorgung erhalten bleibt. Im übrigen ist die schrittweise Erhöhung des Pensionseintrittsalters auf das 67. Lebensjahr geplant.

In Frankreich wird bis zum Jahr 2020 eine weitgehende Harmonisierung zwischen dem allgemeinen System und den systèmes alignés einerseits und dem des öffentlichen Dienstes andererseits angestrebt. Auf diesem Weg wurden einige Besonderheiten des Systems der Beamtenalterssicherung bereits abgeschafft, andere werden in Zukunft beseitigt werden. Damit wird das System der Beamten immer mehr dem allgemeinen Sicherungssystem angeglichen werden, ein Prozeß der Harmonisierung dem rechtlich kaum Hindernisse entgegen stehen. Allerdings deutet gegenwärtig wenig darauf hin, daß das Beitragssystem des régime général für das Sondersystem übernommen werden 
oder die Leistungshöhe explizit vermindert werden soll. Als Teil der Annäherung der Systeme werden frei werdende Beamtenstellen zunehmend nicht mehr mit Beamten, sondern mit Arbeitnehmern besetzt.

Italien hat den Reformprozeß zur Angleichung des Sondersystems für Beamte an die Regelungen der Rentenversicherung für die Arbeitnehmer der Privatwirtschaft weitgehend bereits hinter sich. Dieser Abbau von Privilegien war sogar eine der Voraussetzungen, die seit den 1990er Jahren erforderlichen Reformen des allgemeinen Systems politisch durchzusetzen.

In Dänemark wurden bestimmte Dysfunktionen des allgemeinen Systems beseitigt. Ab 2009 soll das Rentenalter schrittweise auf 67 angehoben werden. Die Pensionsordnungen der Beamten blieben unverändert. Hier sind durch den Abbau von Beamtendienststellen und durch deren Verlagerung zum allgemeinen öffentlichen Dienst Einsparungen zu erwarten.

In Norwegen wurde zum 1. Januar 2010 eine grundlegende Reform des Alterssicherungssystems durchgeführt. Die Bindung der Beamtenpensionen an 2/3 des letzten Gehalts soll jedoch erhalten bleiben. Der Einbau eines demographischen Faktors in die Pensionsformel soll von den Tarifpartnern erarbeitet werden.

Schweden blickt auf eine grundlegende Reform des gesamten Alterssicherungssystems zurück. Die Beamtenversorgung ist dadurch nur hinsichtlich ihres beitragsbezogenen Teils betroffen: Hier wurde nach dem Beispiel der „Prämienrente“ des allgemeinen Systems den Beamten die Möglichkeit gegeben, die Beiträge auf dem Kapitalmarkt anzulegen. Beim leistungsbezogenen Teil wurde die Anbindung der Pension an das letzte Durchschnittsgehalt beibehalten.

In der Schweiz wurden grundlegende Reformen des Alterssicherungssystems des Bundespersonals seit den 1990er Jahren durchgeführt. Eine über dem allgemeinen Niveau liegende Alterssicherung des Bundespersonals durch überobligatorische Leistungen des Dienstherrn sowie die faktische Bestimmung der Pensionen durch die letzten Bezüge vor dem Ruhestand blieben dabei bis heute teilweise bestehen.

Die Reform der Zusatzsicherung der civil servants im Vereinigten Königreich durch „nuvos“ wurde bereits erwähnt. Dabei hielt man am Charakter eines defined benefit system fest. Zudem soll die Option, die einkommensbezogene staatliche Zusatzrente (S2P) zu verlassen, nur noch auf der Basis von defined benefits und nicht mehr von defined contributions möglich sein. Aktuell wird an einer gesetzlichen Grundlage für die Ausgestaltung des Civil Service gearbeitet. Inhaltliche Änderungen sind dabei aber zunächst nicht zu erwarten.

Für die Russische Föderation sind diverse Reformprozesse zu konstatieren. Zum 1. Januar 2010 soll etwa die Finanzierung der Rentenversicherung auf ein reines Beitragssystem umgestellt werden. Es ist gegenwärtig nicht abzusehen, ob dadurch und durch andere zu erwartende Reformen der Leistungsabstand des Versorgungsniveaus der Pensionen der Staatsbediensteten zur allgemeinen Arbeitsrente erhalten bleibt. 
Die Alterssicherungssysteme von Slowenien und der Tschechischen Republik sind im europäischen Vergleich jung. Das derzeit geltende, auch die Staatsbediensteten einschließende Rentenversicherungsrecht wird deshalb in naher Zukunft zwar wohl nicht ohne Modifikationen bleiben, tiefgreifende Reformen sind jedoch nicht zu erwarten.

\section{Die Rechtslage in den Internationalen Organisationen des VN-Systems und der EU}

\section{Das Beamtenstatut der ,,internationalen Beamten“}

Das EU-Beamtenstatut ist in seiner Regelungstiefe und Komplexität dem deutschen Beamtenrecht vergleichbar. Es beruht auf der in Art. 283 EGV enthaltenen Kompetenzregelung. Beamter der EU ist, wer bei einem ihrer Organe durch Urkunde nach den Vorschriften des Statuts unter Einweisung in eine Dauerplanstelle zum Beamten ernannt worden ist. Im Statut ist die Erfüllung einer Reihe weiterer persönlicher Voraussetzungen vorgeschrieben. Es nennt auch spezifisch beamtenrechtliche Pflichten, die von der Loyalität zum Dienstherrn, Verschwiegenheit und Unparteilichkeit bis hin zur Residenzpflicht am Dienstort reichen. Dem korrespondiert eine vom EuGH bestätigte, besondere Beistands- und Fürsorgepflicht des Dienstherrn gegenüber den Beamten. Diese umfaßt das Recht auf Besoldung, soziale Sicherheit und Versorgung.

Das Dienstrecht der internationalen Beamten des VN-Organisationensystems folgt den Vorgaben der VN-Charta. Die darin angeführten Pflichten und Einstellungsvoraussetzungen werden vom Personalstatut übernommen und weiter ausgeführt. Das Dienstverhältnis gilt als „Beschäftigungsverhältnis sui generis“. Im Statut finden sich aber zahlreiche Parallelen zum traditionellen Beamtenrecht: Gelöbnis, Loyalitätspflicht, Verschwiegenheitspflicht, Genehmigungsvorbehalt für Nebenbeschäftigungen etc. Auch eine Disziplinarordnung wurde erlassen. Mit diesen Pflichten korrespondieren weitgehende, vom Verwaltungsgericht der Vereinten Nationen im Einzelnen definierte Rechte der Beamten auf Fürsorge durch die jeweilige (Sonder-) Organisation, darunter die Mitgliedschaft im „Gemeinsamen Pensionsfonds“ der Vereinten Nationen.

Die Bediensteten aller Internationalen Organisationen tragen, bei einzelnen Unterschieden, die Bezeichnung ,internationale Beamte“ nicht umsonst. Sie genießen eine von den Organisationen abgeleitete, an ihrer Funktion ausgerichtete Immunität. Ihr Dienstverhältnis beruht durchweg auf einem Personalstatut, das besondere Rechte und Pflichten statuiert und damit einen Sonderstatus rechtlich begründet. 


\section{Altersversorgung}

Die Alterversorgung der Beamten der Europäischen Union sieht vor, daß nach einer Wartezeit von zehn Dienstjahren ein leistungsorientiertes Altersruhegeld von höchstens $70 \%$ des letzten Grundgehalts der letzten Besoldungsgruppe, in die der Beamte mindestens ein Jahr lang eingruppiert gewesen ist, als monatliche Rente ausgezahlt wird. In jedem Dienstjahr werden Anwartschaften auf 1,9\% dieses letzten Grundgehalts erworben. Das Ruhestandsalter ist nach der Reform nicht mehr das 60., sondern das vollendete 63. Lebensjahr. Es besteht eine Mindestgarantie zur Sicherung des Existenzminimums. Die Versorgungsbezüge sind bei Beschlüssen des Rates über die Dienstbezüge an diese gekoppelt. Beim Ausscheiden vor dem Pensionsalter ohne Anspruch auf Ruhegehalt hat der Beamte Anspruch auf ein Abgangsgeld. Das Versorgungssystem wird anteilig aus dem Haushalt und von den Beamten selbst finanziert. Diese tragen zu einem Drittel der Finanzierung durch Beiträge in Höhe von nunmehr 10,25 \% des Grundgehalts bei. Das Gleichgewicht des Versorgungssystems wird dadurch gesichert, daß im Abstand von fünf Jahren versicherungsmathematische Bewertungen durchgeführt werden, nach deren Ergebnissen Beitragssatz und Ruhestandsalter verändert werden können. Dem europäischen Gesetzgeber kommt bei der Ausgestaltung des Versorgungssystems im Hinblick auf die Wahrung des Haushaltsgleichgewichts nach der Rechtsprechung der EU-Gerichte ein weiter Ermessenspielraum zu.

Nach der Pensionsordnung des „Gemeinsamen Pensionsfonds“ der Vereinten Nationen wird eine leistungsbezogene Alterspension von maximal $70 \%$ des letzten Durchschnittsgehalts des Beamten gezahlt, wobei die Beitragsjahre unterschiedlich bewertet werden. Auf Antrag der Beamten besteht die Möglichkeit, geringfügige Pensionsansprüche sowie Ansprüche über einer bestimmten Höhe ganz oder teilweise (bis höchstens 1/3 der Anwartschaft) als ,lump sum“ auszuzahlen. Für Notsituationen sind besondere Hilfen vorgesehen. Die Finanzierung beruht auf Beiträgen der Beamten und der internationalen Organisation im Verhältnis 1:2, derzeit 7,9\% bzw. 15,8 \% des jeweiligen Gehalts. Dazu kommen Gewinne aus der Vermögensanlage des Fonds.

\section{Reformen}

Die beamtenrechtliche Struktur der Altersversorgung der EU-Beamten steht nicht zur Disposition. Allerdings wurde durch die Reform des Jahres 2004 das Ruhestandsalter von 60 auf 63 Jahre angehoben und der individuelle Beitrag der Beamten erhöht. Die Reformschritte sind jedoch moderat ausgefallen. Weitere Reformen liegen aufgrund des steigenden Kostendrucks des EU-Pensionssystems nahe. Ein radikaler Umbau des EUBeamtenrechts ist aber weder geplant und wäre wohl auch kaum mit den gegenwärtigen rechtlichen Vorgaben zu vereinbaren. 
Trotz erfolgreicher Fondsverwaltung erwiesen sich auch für den „Gemeinsamen Pensionsfonds" der Vereinten Nationen Einsparmaßnahmen als unumgänglich. Dabei wurde bislang von der Durchsetzung der Verpflichtung der Mitgliedsorganisationen, gegebenenfalls die Fondsmittel aufzustocken, allerdings bisher abgesehen. Statt dessen kam es zu der Anhebung der Altersgrenze der Beamten auf 62 Jahre, der Verlangsamung des prozentualen Erreichens des höchsten Pensionssatzes und der Anpassung laufender Pensionen, der Erhöhung der Beiträge der Bediensteten um 0,9 \%, der der Mitgliedsorganisationen um 1,8 \%, sowie dem Abbau der Steuererstattung im Fall der Kapitalisierung der Pension. Die Rechtsprechung des Verwaltungsgerichts der VN bestätigte notwendige Anpassungen der Pensionsordnung zwar über die Jahre immer wieder, im Hinblick auf wohl erworbene Rechte der Bediensteten wurden dafür aber enge Grenzen gezogen.

\section{Auswertung}

\section{Beamtentum als Institution des Verfassungsrechts}

Als erstes kann bei einem aktuellen Vergleich des deutschen Beamtenrechts mit dem Recht von zehn europäischen Ländern und mit dem für ein System internationaler Organisationen geltenden Dienstrecht sowie dem einer supranationalen Organisation nur aufs Neue bestätigt werden, was Tomandl schon vor ziemlich genau 30 Jahren festgestellt hat: Unter den Vergleichsländern kennt nur die Bundesrepublik Deutschland eine Verfassungsgarantie des Berufsbeamtentums, „die jeder grundlegenden Umgestaltungsabsicht deutliche Schranken setzt." 1

Zwar findet der Staatsdienst mit Ausnahme des Vereinigten Königreichs und der Tschechischen Republik in den Verfassungen aller ${ }^{2}$ Vergleichsländer Erwähnung doch nirgendwo als institutionelle Garantie. In den jungen Demokratien der Russischen Föderation, der Tschechischen Republik und Sloweniens regelt die Verfassung, wenn überhaupt, für den Staatsdienst im wesentlichen nur die Gewährleistung gleicher $\mathrm{Zu}$ gangsmöglichkeit für alle Bürger.

1 Tomandl, Generalbericht, in: Zacher/Bullinger/Igl (Hrsg.), Soziale Sicherheit im öffentlichen Dienst in der Bundesrepublik Deutschland, den Niederlanden, Schweden, Belgien und den Europäischen Gemeinschaften, 1982. Zur Arbeitsgruppe der GFR siehe Köhler, Hintergrund: Erfahrungen des Rechtsvergleichs und verfassungsrechtliche Vorgaben in Deutschland, in diesem Band, S. $27 \mathrm{ff}$.

2 In der Schweizer Verfassung vom 18. April 1999 (Stand am 17.5.2009) wird in Art. 35 Abs. 2 (Verwirklichung der Grundrechte) die Verwaltung auf die Grundrechte verpflichtet: „Wer staatliche Aufgaben wahrnimmt, ist an die Grundrechte gebunden und verpflichtet, zu ihrer Verwirklichung beizutragen.“ In Art. 178 Abs. 3 (Bundesverwaltung) die Möglichkeit eröffnet, Verwaltungsaufgaben auch Privaten zu übertragen: „Verwaltungsaufgaben können durch Gesetz Organisationen und Personen des öffentlichen oder des privaten Rechts übertragen werden, die außerhalb der Bundesverwaltung stehen.“. 
Die verfassungsrechtliche Seite des Vergleichs kann deshalb so zusammengefaßt werden: Obwohl das Grundgesetz der Bundesrepublik Deutschland in den Ländern Europas durchweg als gelungene und inzwischen auch als bewährte moderne Verfassung gewürdigt wird, gab es für die Normierung der ,hergebrachten Grundsätze des Berufsbeamtentums“ und der vom Bundesverfassungsgericht daraus entwickelten „grundrechtsgleichen Position" des Beamtentums keine Nachahmer.

Es muss hier nicht geklärt werden, ob dies daran liegt, daß diese Position sich einer womöglich spezifisch deutschen Tradition verdankt, die es eben andernorts so nicht gibt - denn auch für die meisten der „,hergebrachten Grundsätze“ selbst finden sich einzelne Parallelen, der gesamte Katalog ist aber in keinem Land und keiner Organisation in das Recht oder die Rechtsprechung übernommen worden. So werden meist nur spezifisch hervorgehobene Beamtenverhältnisses zwingend durch Gesetz geregelt, eine Einstellung auf Lebenszeit ist generell die Ausnahme, das Laufbahnprinzip findet eigentlich nur in Frankreich eine freilich keineswegs deckungsgleiche Parallele und das Streikverbot gilt nirgendwo so strikt wie in Deutschland.

\section{Alimentation und Alterssicherung}

Das im Zusammenhang mit der Beamtenversorgung in Deutschland primär herangezogene Alimentationsprinzip belegt ebenfalls vor allem das Vorliegen eines ,deutschen Sonderwegs": Aus Sicht des Bundesverfassungsgerichts ${ }^{3}$ gehört es zum Katalog der wesentlichen hergebrachten Grundsätzen des Berufsbeamtentums. Es verpflichtet den Dienstherrn insbesondere, den amtsangemessenen Unterhalt - das bedeutet nach deutschem Recht Besoldung und Versorgung - des Beamten und seiner Familie sicherzustellen. Nach dem Bundesverfassungsgericht gehört zur Alimentation auch, daß die Beamten nicht selbst Beiträge zur Finanzierung ihrer Versorgung im Ruhestand leisten müssen und, damit die Versorgung auch amtsangemessen bleibt, deren Höhe vom Gehalt der letzten zwei Dienstjahre bestimmt wird.

3 Das ist weder in der Wissenschaft noch bei den Beamten selbst unumstritten: So weist Summer, Alimentationsprinzip gestern und heute, ZBR 1981, S. 1 ff. - abgedruckt auch in: Pechstein (Hrsg.), Rudolf Summer, Beiträge zum Beamtenrecht, 2007, S. 1 ff., schon 1981 darauf hin, daß die Beamten selbst die Besoldung als Gegenleistung für ihren Dienst sehen, ,sie wollen keine ,Alimente' erhalten. Mit eine Ursache für die Skepsis ist, daß das Alimentationsprinzip in der beamtenpolitischen Entwicklung der Nachkriegszeit weit mehr zur Abwehr von Ansprüchen als zur Begründung von Ansprüchen herangezogen wurde." Summer resümiert dann, daß der Begriff Alimentation nicht richtig kennzeichnet, was damit gemeint ist, „die Schwierigkeit besteht jedoch darin, hierfür etwas anderes zu setzen (S. 40). 
Es zeigt sich, daß in allen Vergleichsländern mit Ausnahme Schwedens ${ }^{4}$ die Staatsbediensteten auf unterschiedliche Weise ebenso Beiträge für ihre Alterssicherung abführen, wie die internationalen Beamten der Europäischen Union und des VN-Systems.

Italien, die Schweiz, Slowenien und die Tschechische Republik kennen keine Anbindung der Ruhestandsbezüge an die Dienstbezüge, und im Vereinigten Königreich ist man dabei, die Fortschreibung der Bezüge in der Altersversorgung abzuschaffen. Andererseits werden in Frankreich (noch) die Pensionen vom Gehalt der letzten sechs Dienstmonate bestimmt, wovon bis zu $75 \%$ erreicht werden können. In Dänemark belaufen sie sich auf $57 \%$ des Letztgehalts, in Norwegen auf $66 \%$ davon, in Schweden wiederum auf den Durchschnitt der letzten fünf Dienstjahre und in der Russischen Föderation gilt der Durchschnitt der letzten zwölf Monate bis zu einer Obergrenze. Die internationalen Beamten der Europäischen Union und der Vereinten Nationen erhalten bis zu $70 \%$ des (EU: im letzten Dienstjahr) durchschnittlich erzielten Gehalts.

Unabhängig vom Abstellen auf eine beamtenrechtliche Begründung zeigt der Vergleich aber, daß (mit Ausnahme der jungen Demokratien Russland, Slowenien und Tschechien) in allen Ländern (und mutatis mutandis gilt dies auch für die Vereinten Nationen und die Europäische Union) die Leistungen der Altersversorgung des Staatsdienstes der Höhe nach (mehr oder weniger und z. T. über „quasibetriebliche“ Zusatzleistungen) über denen der allgemeinen Systeme der Alterssicherung liegen. Zugleich wird aber eine Tendenz dahin erkennbar, daß diese Vorteile, gewöhnlich Relikte des in der Vergangenheit hervorgehobenen Status der Beamtenschaft, in den verschiedenen Staaten vielleicht in unterschiedlichem Tempo abgebaut, nirgends aber gestärkt oder gar neu eingeführt wurden.

Dieser Prozeß des Abbaus überkommener Privilegien verlief in vieler Hinsicht parallel: Zum einen erfolgte und erfolgt er in den Vergleichsländern in für die Betroffenen zumutbaren, Vertrauensschutz, Rückwirkungsverbot und wohl erworbene Rechte beachtenden Schritten. Die auch bei den Vereinten Nationen und der Europäischen Union notwendigen Einsparungen bei der Altersversorgung setzten auf diese Weise am Ruhestandsalter und an den Beiträgen an, tiefgreifende Änderungen der Systeme konnten aber bisher weitgehend vermieden werden. Zum anderen wurde der demografische und ökonomische Druck auf die Versorgungssysteme dadurch gemindert, daß immer größere Sektoren des öffentlichen Dienstes nicht mehr mit Beamten, sondern mit Angestellten besetzt wurden und werden.

4 Was aber keine beamtenspezifische Ursache hat, sondern daher rührt, daß über Jahrzehnte in Schweden die Finanzierung der gesamten sozialen Sicherheit ausschließlich über allgemeine Steuern oder Arbeitgeberbeiträge erfolgte. 


\section{Die Wahrnehmung hoheitlicher Aufgaben}

Ob vom jeweiligen Gesetzgeber bewußt gesetzt oder nicht, die Reformen des öffentlichen Dienstes in den Vergleichsländern via Reduzierung der Beamtenschaft zeigen, daß dabei, wie in Deutschland durch Art. 33 Abs. 4 GG vorgegeben und europarechtlich durch die Rechtsprechung des EuGH, auf eine unmittelbare oder mittelbare Teilnahme an der Ausübung hoheitlicher Befugnisse und an der Wahrnehmung solcher Aufgaben abgestellt wird, die auf die Wahrung der allgemeinen Belange des Staates gerichtet sind.

Vor der Antwort auf die Frage, ob aus der Besonderheit des Dienstherrn (Staat) und der Aufgaben (Amt, hoheitlich) eine Besonderheit der Versorgung folgt, ist deshalb zu klären, ob es rechtliche Grenzen für den faktischen Abbau der Beamtenschaft gibt, ob also die „Ausübung hoheitsrechtlicher Befugnisse“ zwingend nur durch Beamte ,in einem öffentlich-rechtlichen Dienst- und Treueverhältnis“ erfolgen darf. Der Rechtsvergleich zeigt, daß diese Frage sich für das Vereinigte Königreich, die Schweiz, Slowenien und Tschechien gar nicht erst stellt und in Deutschland wohl vor allem deshalb einer anhaltenden Diskussion unterliegt, weil es nur hier (neben einer traditionell politisch einflussreichen Beamtenlobby) die verfassungsrechtliche Garantie des Berufsbeamtentums besteht und ein diese konsequent stützendes Verfassungsgericht.

Die Schwierigkeit, „hoheitsrechtliche Aufgaben“ überzeugend allgemeingültig zu definieren, besteht weiter. Im Grunde wird das solange so sein, wie man daran festhält, sowohl die Eingriffsverwaltung wie auch die Leistungsverwaltung als hoheitliche Tätigkeit zu verstehen. ${ }^{5}$ Dabei kennt die Praxis etwa im Bereich der Daseinsvorsorge auch in Deutschland längst die Übernahmen solcher Funktionen durch Private, was „die faktischen Grenzen des Erfordernisses eines Einsatzes von Beamten"6 aufzeigt. Die Rechtslage in den Vergleichsländern bestätigt, daß moderne Staaten effiziente Leistungsverwaltungen durchaus auch ganz ohne Beamte durchführen können. Wenn es etwa für Dänemark heißt, daß bald nur mehr „Bedienstete in Uniform“ verbeamtet sein werden, so scheint dies auch für die anderen Vergleichsländer, wenn nicht schon Gegenwart, so doch die Zukunft zu sein.

5 Reich, Beamtenstatusgesetz, Kommentar, 2009, Rdnr. 6 zu $§ 3$ Abs. 2 Ziff. 1 BeamtStG findet sich dafür notgedrungen die wenig hilfreichen Unterscheidungen nach „Hoheitsverwaltung im engeren Sinn“ und „schlichte Hoheitsverwaltung“. Leisner, „Privatisierung“ des Öffentlichen Rechts, Von der „Hoheitsgewalt“ zum gleichordnenden Privatrecht, 2007, S. 100, geht weiter: „Doch daß es überhaupt und wo es ,hoheitliche Befugnisse' geben müsse, legt eben Art. 33 Abs. 5 GG nicht näher mit Verfassungskraft fest.“

6 Ders., ebda. 
4. Ein besonderes Beamtenethos gilt nur für besondere Funktionen - „Das Amt macht den Beamten "

Die hier verglichenen Länder bieten keinen Anhaltspunkt dafür, daß etwa aufgrund unbefriedigender Erfahrungen mit der Privatisierung der Durchführung öffentlicher Aufgaben oder mit dem besser klingenden new public management mittlerweile „eine Rückbesinnung auf das öffentliche Amt und auf den am Gemeinwohl ausgerichteten Amtswalter festzustellen sei“ 7 - in der Verbindung von „Amt“ mit der Bezeichnung „Amtswalter" scheint aber eine andere Rückbesinnung auf: Was sich aus der Etymologie $^{8}$ ableiten läßt, wird so zum auch aktuell hilfreichen Entscheidungskriterium bei der Definition: Erst das Amt „macht“ den Beamten.

Dies wird besonders bei den Internationalen Beamten deutlich. Eine der wesentlichen Funktionsvoraussetzung für internationale und erst Recht für supranationale Organisationen ist die über singulären nationalen Interessen der Mitgliedstaaten agierende Neutralität. Das darauf beruhende Selbstverständnis der Organisationen erlaubt es, von ihren Beamten, die ja national rekrutiert werden, eine besondere Loyalität zu fordern, ein spezifisches, dem Völkerrecht verpflichtetes Beamtenethos ${ }^{9}$. Dieses wegen seines internationalen Bezugs ,besonders besondere“ Dienst- und Treueverhältnis soll kein beliebiger „Job“ sein. Es verlangt den Beamten spezifische Pflichten ab, die etwa über die üblichen arbeitsvertraglichen Loyalitätspflichten hinausgehen.

Da dies auch damit zusammenhängt, daß für die Position der internationalen Beamten mutatis mutandis noch der in den nationalen Rechtsordnungen inzwischen nicht mehr enthaltene Aspekt der Repräsentation des Dienstherrn gilt, kann man einwenden, diese Art Beamtenethos sei ein Ausnahmefall und im modernen Dienstleistungsstaat weithin von der Entwicklung überholt.

Das Bundesverfassungsgericht teilt diese Zweifel freilich nicht. Vor kurzem ist die Verfassungsbeschwerde eines Justizvollzugsobersekretär gegen die Anordnung des Leiters der Justizvollzugsanstalt, jede Dienstunfähigkeit infolge Krankheit durch unverzügliche Vorlage eines privatärztlichen Attests spätestens an dem der Erkrankung folgenden ersten Arbeitstag nachzuweisen, nicht zur Entscheidung angenommen. Der Beschluß ist deshalb bemerkenswert, weil die Entscheidung einer an sich einfachen ar-

7 Ders., ebda.

8 S. Grimm/Grimm, Deutsches Wörterbuch, 16 Bde. [in 32 Teilbänden], 1854-1960, Lemma Beamte. Danach stand am Anfang das „Amt“, wer damit betraut war, (=munere fugens) wurde mit dem davon abgeleiteten Partizip als „beampt“ bezeichnet, der Beamte definiert sich also über das ausgeübte Amt. Der Zusammenhang gilt auch im Französischen „fonction“/,fonctionnaire“, im sprachverwandten Italienischen und ähnlich auch im Russischen, vgl. Chesalina, Die Altersversorgung von Beamten in der Russischen Föderation, in diesem Band, S. 133 ff.

9 Im VN-System wird dies u.a. durch die Vorgabe eines schriftlich abzulegenden feierlichen Dienstgelöbnisses auf die besonderen Pflichten als internationale Beamte deutlich. Siehe Köhler, Die Altersversorgung der internationalen Beamten nach dem Dienstrecht der Vereinten Nationen, II. Personalstatut, in diesem Band, S. $235 \mathrm{ff}$. 
beitsrechtlichen Frage gilt, das Gericht es aber dennoch für geboten richtig hält, zur Begründung u.a. auf hohe persönliche Verpflichtungen, auf das Ethos des Berufsbeamtentums und auf die unbedingte Loyalität gegenüber dem Rechtsstaat zu verweisen:

\begin{abstract}
„Mit der Berufung in das Beamtenverhältnis ist die Pflicht des Beamten verbunden, seine ganze Persönlichkeit für den Dienstherrn einzusetzen und diesem seine volle Arbeitskraft zur Verfügung zu stellen (vgl. BVerfGE 55, 207, 236 f.; 61, 43, 56; 70, 69, 80). Das Amt erfordert ein Ethos, das gekennzeichnet ist durch ein die gesamte Persönlichkeit prägendes hohes Maß an Verantwortungs- und Pflichtbewusstsein, unbedingte Loyalität gegenüber dem Rechtsstaat, aktives Eintreten für diesen Staat, die Zurücknahme privater Bedürfnisse und Empfindlichkeiten zugunsten des Amtes sowie dessen würdevolle Ausübung." 10
\end{abstract}

In der Literatur finden sich so rigorose Sätze nur mehr selten. Mehr Zustimmung findet die Ansicht, daß nicht allein der Beschäftigungsstatus, sondern die Verwaltungskultur insgesamt dafür entscheidend ist, inwieweit das Verwaltungspersonal rechtsordnungsgemäß verfährt. ${ }^{11}$ Rein tatsächlich gilt eine „ethisch-verklärte Betrachtung des Beamtentums" als kaum noch vermittelbar. 12

Im Vereinigten Königreich, in der Schweiz, in Italien und den jungen Demokratien im Osten Europas, aber auch in Frankreich ist diese die Realitäten des dienstlichen Alltags verklärende Betrachtungsweise an sich unbekannt. Die Nordischen Länder könnten insofern über die Region hinaus beispielhaft sein, weil dort der Beamtenstatus tatsächlich in dem Sinn an das Amt gebunden ist - als eben nicht jede Beschäftigung im öffentlichen Dienst die Hervorhebung erhält, die dort nur für ganz besondere Funktionen demokratisch als vertretbar gilt.

\title{
5. Ergebnis
}

Ein weiterer Ertrag des Rechtsvergleichs scheint bemerkenswert: In keinem der Vergleichsländer gab es so viele Reformansätze im Beamtenrecht mit so minimalen Ergebnissen zu verzeichnen wie in Deutschland. Auf die (zum Teil ja auch im Regierungsauftrag erstellten) rechtsvergleichenden Arbeiten der 1970er und 1980er Jahre wurde in

10 BVerfG, Beschluß vom 28.9.2007, 2 BvR 1156/06. Danach war für das Gericht „nicht ersichtlich, wie der Beschwerdeführer durch die Pflicht, seine Dienstunfähigkeit am nächsten Arbeitstag durch ärztliche Atteste nachzuweisen, in seiner Menschenwürde verletzt sein könnte. Erhöhte Anforderungen an den Nachweis der Dienstunfähigkeit, für die sich der Dienstherr auf konkrete Umstände stützen konnte, erniedrigen einen Beamten nicht.“ Hätte diese einfache Feststellung die Entscheidung nicht auch getragen?

11 Bull, Positionen, Interessen und Argumente im Streit um das öffentliche Dienstrecht, Die Verwaltung 2004, S. 327, 344. Ausdrücklich zustimmend Remmert, Warum muss es Beamte geben?, JZ 2005, S. 53, 59.

12 Bull, Leistungsorientierte Bezahlung im öffentlichen Dienst - Probleme und Lösungsansätze, in: Magiera u.a. (Hrsg.), Verwaltungswissenschaft und Verwaltungspraxis in nationaler und transnationaler Perspektive, Festschrift für Heinrich Siedentopf zum 70. Geburtstag, 2008, S. 531, 533. 
diesem Band bereits hingewiesen ${ }^{13}$. Zwischenzeitlich wären konkrete Änderungsvorschläge zu Art. 33 Abs. 5 GG durch das Land Schleswig-Holstein (1996) ${ }^{14}$, Bestrebungen im Rahmen der Arbeit der Föderalismuskommission (2003/2004), ${ }^{15}$ oder durch das Land Nordrhein-Westfalen (2003) ${ }^{16}$ hervorzuheben. In den Beratungen der Föderalismuskommission wurden wieder die Meinungen mit den je zugehörigen Argumenten zu Art. 33 Abs. 4 und Abs. 5 GG vorgetragen, die seit Jahrzehnten in der Diskussion stehen. ${ }^{17}$ Inzwischen werden sie schon fast gewohnheitsmäßig nach ihren vorweg ja bekannten Erfolgschancen abgestuft eingeordnet. So gilt etwa der Vorschlag einer ersatzlosen Streichung des Art. 33 Abs. 5 GG als „,von vorneherein ohne Erfolgschance“18. Die schließlich in Art. 33 Abs. 5 GG eingefügte Fortentwicklungsklausel reflektiert deshalb auch nicht mehr als „die Absicht des Gesetzgebers, den Verfassungstext, kaum aber die Verfassungsrechtslage zu ändern."19

Die von der Landesregierung Nordrhein-Westfalen eingesetzte Kommission schlug zur Vereinheitlichung des öffentlichen Dienstrechts u.a. - erfolglos - die Streichung des Art. 33 Abs. 5 GG vor. ${ }^{20}$

Für die hier gestellte Frage nach der „Verknüpfung von Vergütung und Versorgung“ wird vorgeschlagen, das System der Altersversorgung im öffentlichen Dienst mit dem des übrigen Beschäftigungssystems zu synchronisieren. Dies soll durch zwei Säulen erfolgen:

- gesetzliche Rentenversicherung (,1. Säule') und

- zusätzliche Versorgung analog zur Betriebsrente (,2. Säule') 21

Mit dieser (gewollten oder ungewollten) Annäherung an die Systeme der Schweiz, des Vereinigten Königreichs und der Nordischen Länder wird (bewußt oder nicht) auch eine die schwedische Rentenreform tragende Hoffnung verbunden, durch die rechtliche und organisatorische Gestaltung des gesamten Systems dieses „dem Zugriff der Politik" ${ }^{\prime 22}$ zu entziehen.

Es gibt keine Anhaltspunkte dafür, daß der Gesetzgeber diesen oder ähnliche Vorschläge übernehmen will, obwohl auch der vorliegende Rechtsvergleich zeigt:

13 Köhler (Fußn. 1), S. 27 ff.

14 Antrag zur Modifizierung des Art. 33 Abs. 5 GG im Bundesrat, BR-Drs. 298/96. Einen Überblick geben Höfling/Burkiczak, Die Garantie der hergebrachten Grundsätze des Berufsbeamtentums unter Fortentwicklungsvorbehalt, Erste Überlegungen zur Änderung von Art. 33 Abs. 5 GG, DÖV 2007, S. $328 \mathrm{ff}$.

$15 \mathrm{Zu}$ deren Arbeit s. dies., ebda., S. 328, $331 \mathrm{ff}$.

16 Bericht der von der Landesregierung Nordrhein-Westfalen eingesetzten Kommission: Zukunft des öffentlichen Dienstes - öffentlicher Dienst der Zukunft, Neuauflage 2004.

17 Mit Nachweisen der Protokolle Höfling/Burkiczak (Fußn. 14), DÖV 2007, S. 328, 332.

18 Dies., a.a.O.

19 Dies., a.a.O.; zu dieser Änderung des Art 33 Abs. 5 GG auch Köhler (Fußn. 1), S. 37 ff.

20 Bericht der „Bull-Kommission“(Fußn. 16), S. 170.

21 Ebda., S. 139.

22 Ebda., S. 23. Gerade nach den Erfahrungen Schwedens muß die Realisierung dieser Hoffnung als „ohne Erfolgschancen“ qualifiziert werden. 
- Besonderheiten des Dienstrechts, aus denen plausibel Besonderheiten der sozialen Sicherung der Bediensteten im Alter abzuleiten wären, finden sich allenfalls im Dienstrecht der Vereinten Nationen und der Europäischen Union,

- in den Vergleichsländern nur mehr marginal

- und, wenn überhaupt, auf wenige funktional hervorgehobene Ämter beschränkt.

Damit läßt sich das Ergebnis des Rechtsvergleichs kurz fassen: Die deutsche Beamtenversorgung steht aufgrund ihrer einfachgesetzlichen Ausgestaltung nach vom Bundesverfassungsgericht strikt ausgelegten verfassungsrechtlichen Vorgaben zumindest im europaweiten Vergleich allein. 